\title{
Bile acids in treatment of ocular disease
}

\author{
Jeffrey H. Boatright • John M. Nickerson • \\ Anisha G. Moring • Machelle T. Pardue
}

Received: 20 July 2009 /Accepted: 31 July 2009/Published online: 27 August 2009

(C) The Author(s) 2009. This article is published with open access at Springerlink.com

\begin{abstract}
Bear bile has been included in Asian pharmacopeias for thousands of years in treatment of several diseases, ranging from sore throat to hemorrhoids. The hydrophilic bile acids tauroursodeoxycholic acid (TUDCA) and ursodeoxycholic acid (UDCA) are the major bile acids of bear bile. Both of these are available as synthetic formulations and are approved by the health administrations of several countries for treatment of cirrhosis and gallstones. This review briefly covers the use of bear bile in Traditional Chinese Medicine, bile acid physiology, approved use of UDCA and TUDCA in Western medicine, and recent research exploring their neuroprotective properties, including in models of ocular disease.
\end{abstract}

Keywords Neuroprotection · Apoptosis ·

Retinal degeneration - Bile acid - Traditional Chinese medicine - Tauroursodeoxycholic acid - TUDCA .

Ursodeoxycholic acid · UDCA

\section{Bear bile use in traditional Chinese medicine}

Bear bile, bile extract, and gallbladder have been used in Traditional Chinese Medicine (TCM) and other Asian pharmacopeia for thousands of years [1-3]. Bear bile is

J. H. Boatright $(\bowtie) \cdot J$. M. Nickerson · A. G. Moring $\cdot$

M. T. Pardue

Department of Ophthalmology,

Emory University School of Medicine,

B5511 Emory Eye Center, 1365-B Clifton Road,

Atlanta, GA 30322, USA

e-mail: jboatri@emory.edu

M. T. Pardue

Rehab R\&D CoE, Atlanta VA Medical Center,

Atlanta, GA, USA classified as a "cold" medicine used to treat diseases of "heat" that involve fevers and inflammation to detoxify the liver, to prevent convulsions and epileptic seizures, to dissolve kidney stones and gallstones, to shrink tumors and hemorrhoids, and to improve vision [1-3]. Its use continues in TCM and has spread with the establishment of Asian communities throughout the world [3]. China's State Pharmacopoeia lists 28 types of medicines containing bear bile, 15 of which are used in ophthalmology [4].

The Asian black bear (Ursus thibetanus or Selenarctos thibetanus), also known as the Moon bear due to its coat markings, is the traditional source species, but as Moon bear populations decline, other species such as the North American black bear are used, often with negative consequences on those populations [5]. To address the issue of declining populations and in recognizing that trafficking in animal parts is a component of their rural economy (dried bear bile has a higher street value than cocaine [6]), controlled bear farming was established in China [2-4]. However, the harvesting of bear parts is considered by many to be cruel $[4,7]$, and it is illegal or greatly restricted in many countries [3-6] (particularly the signatory nations of the Convention on International Trade in Endangered Species of Wild Fauna and Flora [8] and additionally in the USA by the Endangered Species Act [9]), and it is not clear that the farming is sustainable economically or politically $[4,7,10]$. Thus, there is great interest in replacing bear parts with herbal and synthetic substitutes in TCM [3, 4].

\section{Bile acid physiology}

Bile acids are biosynthesized from cholesterol in liver hepatocytes. They are largely contained in the enterohepatic 
circulation and are stored in the gallbladder. They are predominantly conjugated with glycine or taurine, allowing them to be fully ionized in the $\mathrm{pH}$ range of the small intestine [11]. Bile acids have five major functions: elimination of cholesterol, micellination of dietary lipids to aid in their transport, stimulation of bile flow and biliary phospholipid secretion, and finally, negative feedback regulation of bile acid and cholesterol synthesis [11].

In humans, the principle primary bile acids are cholic acid and chenodeoxycholic acid (CDCA) and the principle secondary bile acids are deoxycholic acid (DCA) and lithocholic acid. Conversely, the biliary bile and the serum of some bears contain high proportions of ursodeoxycholic acid (UDCA), mainly occurring as its taurine conjugate tauroursodeoxycholic acid (TUDCA) [12-15]. UDCA and conjugates comprise about $47 \%$ of the biliary bile make up in American black bear and up to $76 \%$ in Asiatic black bear [13]. UDCA and TUDCA are found only in trace amounts $(<1-5 \%$ of total biliary bile acid composition) in other vertebrates [13]. Similar to biliary bile content, the taurineconjugated form of UDCA comprises a large proportion (about 28\%) of the circulating plasma levels of bile acids in American black bears [14]. In comparison, TUDCA constitutes about $0.13 \%$ of the bile acid pool in human serum [16]. As UDCA and TUDCA are the major constituents of bile from Asian black bears, these two hydrophilic bile acids have long been considered by many to be the bioactive components of bear bile $[3,4,7,10,14]$.

\section{Synthetic UDCA and TUDCA as therapeutics}

UDCA and TUDCA were first synthesized from cholic acid in 1954 in Japan [17]. Today UDCA is produced by several manufacturers worldwide and marketed under various trade names, including Ursodiol, Actigall, Ursosan, Urso, Urso Forte, and Deursil. UDCA is approved by several governments for treatment of liver diseases and for the dissolution of gallstones. In Italy, TUDCA is marketed as Taurolite and is used similarly. Typical oral dosing of UDCA is $7-15 \mathrm{mg} /$ $\mathrm{kg}$ body weight daily. Both TUDCA and UDCA are well tolerated; the most common side effect is diarrhea in less than $2 \%$ of several populations tested [18-20].

Oral administration of UDCA results in UDCA and its conjugates becoming the proportionately dominant bile acids in biliary bile, and serum and plasma absolute concentrations increase over tenfold [21-26]. About half of the dose is absorbed from the portal blood into liver via first pass extraction, where it is conjugated with glycine forming glycoursodeoxycholic acid (GUDCA) or taurine forming TUDCA [24, 27-29]. The percentage absorbed decreases with increasing dose such that absolute and proportional enrichment of the biliary bile with UDCA and conjugates plateaus at an as-yet undefined dose due to epimerization of UDCA to CDCA and endogenous bile acid synthesis [23, 27, 30, 31]. UDCA and conjugates are excreted from the biliary tree and resorbed through the enterohepatic circulation or metabolized to insoluble salts and excreted in the feces [24]. Oral dosing with TUDCA produces similar changes in biliary bile acid composition and circulating bile acid composition and concentrations, but with higher proportions and concentrations of UDCA and conjugates, possibly due to reduced intestinal biotransformation of TUDCA, suggesting moderately enhanced bioavailability [18, 32].

The initial use of UDCA was for dissolution of gallstones; it dissolves gallstones by solubilizing cholesterol from the stone surface, and it reduces the saturation of cholesterol in the bile, enhancing cholesterol transport [24, $26,33]$. UDCA is still prescribed for gallstone dissolution in patients who cannot or do not want to undergo surgery $[24,27,29]$.

The other major, institutionally approved use of UDCA or TUDCA is in treatment of cholestatic liver disease, especially primary biliary cirrhosis (PBC). The first report of the use of UDCA or TUDCA in treatment of liver diseases in Japanese patients came shortly after their laboratory synthesis was developed in the mid-1950s [24, 29, 34]. However, their use in treatment of liver disease increased greatly after it was fortuitously discovered that serum aminotransferase levels improved in patients with chronic active hepatitis who were receiving UDCA for gallstone dissolution [24, 35], a discovery that sparked a series of studies examining its efficacy in treatment of cholestatic liver disease [11, 24].

\section{UDCA and TUDCA as antiapoptotic cytoprotectants}

TUDCA and UDCA were originally thought to act largely through displacement of hepatotoxic, hydrophobic bile acids such as CDCA and DCA from the bile acid pool, thus preventing accumulation of toxic levels in enterohepatic circulation and so reducing the detergent properties of bile at the hepatocellular membrane [11, 24]. However, two groups nearly simultaneously discovered that UDCA may have more mechanistic actions than simply replacing toxic bile acids. Koga et al. [36] found that biliary epithelial cells (BECs) from PBC patient biopsy tissue had much less DNA fragmentation from patients treated with UDCA than from untreated patients, suggesting that BECs from treated patients undergo less apoptosis than those from untreated patients.

Rodrigues et al. [37] more directly tested for an antiapoptotic mechanism. They isolated mitochondria from rat liver and treated them with UDCA or DCA, a 
hydrophobic bile acid known to be cytotoxic. DCA increased mitochondrial swelling, calcein release, and reactive oxygen species (ROS) production, and decreased the mitochondrial membrane potential. UDCA treatment inhibited these effects. In the same study, they found that rats fed with high DCA diets had high concentrations of Bax protein in their mitochondria, an effect blocked by adding UDCA to their drinking water. This same group then demonstrated that UDCA treatment inhibited apoptosis induced by deoxycholic acid, okadaic acid, and transforming growth factor betal in rat hepatocytes in primary culture [38]. They found that incubation with UDCA prevented cyctochrome $c$ release, caspase activation, and cleavage of poly(ADP-ribose) polymerase. They also found that the apoptosis-inducing agents decreased mitochondrial transmembrane potential (MTP) and increased Bax translocation from the cytosol to the mitochondrial membrane, effects blocked by UDCA. From these combined studies, the authors concluded that UDCA treatment can directly inhibit changes in MTP and ROS production, thus preventing megapore opening, membrane potential transition, and subsequent initiation of an apoptotic cascade [37, 38].

In later experiments, the same group found that exposing isolated mitochondria to purified, recombinant Bax protein induced cyctochrome $c$ release without permeability changes that result in mitochondrial swelling, that is, independently of the permeability transition pore opening. This effect in isolated mitochondria was prevented by coor pre-incubation with TUDCA. Pre-incubating mitochondria with TUDCA, then washing out the media and replacing with fresh, produced the same results, indicating that the effect of TUDCA is not due to direct interaction between TUDCA and Bax protein in the incubation media [39]. Thus, both UDCA and TUDCA have antiapoptotic effects on isolated mitochondria.

More recently, this group and others [40, 41] have demonstrated that in addition to effects at the mitochondrial membrane, UDCA and TUDCA may have antiapoptotic effects by binding or otherwise activating nuclear steroid receptors. Following nuclear translocation, the hydrophilic bile acids appear to modulate the E2F-1/p53/Bax pathway as part of their antiapoptotic mechanism of action (reviewed in $[42,43])$.

\section{UDCA and TUDCA as neuroprotectants}

Steer, Rodrigues, Kren, Low, and colleagues extended their studies in liver disease models to test the effects of UDCA or TUDCA treatment in models of neuronal disease and injury. Treatment of rat striatum in vivo or neuronal cells in culture with 3-nitropropionic acid (3-NP), an irreversible inhibitor of succinate dehydrogenase, induces apoptosis and is considered a model of Huntington's disease (HD). Coincubation of an immortalized rat neuronal cell line or rat striatal cells in primary culture with TUDCA, UDCA, or GUDCA prevented 3-NP-induced apoptotic events such as MTP depolarization, mitochondrial-associated Bax translocation, and mitochondrial release of cytochrome $c[44,45]$. Intraperotineal injection of rats with 3-NP caused loss of striatal volume, mitochondrial swelling, increased TUNEL signal in striatal tissue sections, and Rota-Rod sensorimotor task and cognitive testing deficits [45]. All of these 3-NPinduced effects were largely or completely prevented for up to 6 months if TUDCA was co-administered with the neurotoxin [45]. The authors tested whether TUDCA is also neuroprotective in a genetic model of HD. R6/2 mice that are transgenic for a causative $\mathrm{CAG} /$ polyglutamine repeat expansion of the human HD gene develop striatal intranuclear inclusions containing the proteins huntingtin and ubiquitin [46] followed by progressive neurological deficits similar to those of HD [47]. IP injections of TUDCA starting at 6 weeks of age reduced striatal atrophy, decreased striatal apoptosis, resulted in fewer and smaller ubiquitinated neuronal intranuclear huntingtin inclusions, and improved locomotor and sensorimotor performance [45]. Thus, systemic TUDCA treatment was found to be protective and antiapoptotic in cultured cells and in vivo in acute models and genetic models of HD.

Similarly, using in vivo cell culture and in vitro approaches, the same core group and colleagues found that treatment with UDCA or TUDCA slowed cell death in several neuronal disease models, including Alzheimer's disease [48-54], Parkinson's disease [55], acute hemorrhagic [56] and acute ischemic stroke [57], and neuronal glutamate toxicity [58].

Other laboratories have found that UDCA and TUDCA are protective in models of neuronal damage or degeneration. For example, incubation with UDCA prevents apoptosis in a model of cisplatin-induced sensory neuropathy, possibly by suppressing p53 accumulation [59]. In an in vivo spinal cord injury model, rats injected systemically with TUDCA showed fewer apoptotic cord cells, less tissue injury, and better hind limb function than untreated control animals [60]. Related, though not neuronal, UDCA and TUDCA are antiapoptotic cytoprotectants in ischemia/ reperfusion and oxidative stress models of liver disease or injury [61-71] and models of heart disease [72-75].

\section{TUDCA as protectant in ocular models}

Genetic models of photoreceptor degeneration

Given the effects demonstrated in several models of neurodegeneration, it was an obvious strategy to test 
whether treatment with TUDCA might have effects in various retinal degeneration models. In our initial experiments, we treated $P d e 6 b^{r d 1}$ (rd1) mice with subcutaneous injections of TUDCA in the same dosing regimen as was done with the rodent neurodegeneration models (e.g., $500 \mathrm{mg} / \mathrm{kg}$ body weight every 3 days). Injections started at postnatal day (P) 9 and continued to P21, at which point animals were killed and retinal cryosections were made. As shown in Fig. 1, vehicle-treated retinas showed the expected near-total loss of outer nuclear layer cells. Conversely, TUDCA-treated retinas had varied morphology, ranging from very little outer nuclear layer (ONL) to thick ONL and in some instances the preservation of what appear to be photoreceptor outer segments. The response to TUDCA treatment varied greatly. TUDCA treatment occasionally resulted in enhanced aand b-wave electroretinography (ERG) amplitudes, and morphological changes were only moderately more consistent. This variability was resolved by starting TUDCA injections of rd1 pups earlier (P6) and with daily injections rather than every 3 days [76].
Degeneration of photoreceptors begins by at least P8 in rd1 mice [77], so treatments in our early experiments possibly started too late to provide consistent protection. To address this, we chose to treat another mouse model, the $P d e 6 b^{r d 10}$ ( $\left.\operatorname{dd10}\right)$ mouse, which became available about the time we were testing treatment in the $\mathrm{rd} 1$ mouse [78]. In this mouse, a missense mutation in PDE6B causes degeneration of rods starting at about P14-16 [79, 80]. ERG amplitudes are large enough to be easily measured through the first month of age, but are never normal, as would be expected in mice harboring a mutation in a visual cycle gene [78]. Systemic TUDCA injections of these mice suppressed apoptosis and greatly slowed loss of photoreceptor number, morphology, and function [81, 82]. In rd10 mice at P18, ONL thickness and nuclei counts are about $50 \%$ of wild type, photoreceptor outer segments are largely degenerated, and ERG a- and b-wave amplitudes about $50 \%$ of wild type [80]. In P18 rd10 mice, subcutaneous injections with TUDCA resulted in the preservation of the number of photoreceptor cells (Fig. 2), ONL thickness, photoreceptor outer segments (Fig. 3), and ERG a- and b-
Fig. 1 Photomicrographs of retinal cryosections from TUDCAtreated rd1 mice. Pde6b ${ }^{r d l}$ (rd1) mice were subcutaneously injected with vehicle or TUDCA $(500 \mathrm{mg} / \mathrm{kg}$ body weight every 3 days). Injections started at postnatal day (P) 9 and continued to P21, at which point animals were killed, and retinal paraffin sections were cut and stained with hematoxylin and eosin (H\&E-stained). Vehicletreated retinas showed the expected near-total loss of ONL cells. Conversely, TUDCAtreated retinas had varied, but more organized morphology ranging from very little ONL to thick ONL and in some instances the preservation of what appear to be photoreceptor outer segments. RPE: retinal pigment epithelium; IS/OS: inner segment/outer segment; $O N L$ : outer nuclear layer; $O P L$ : outer plexiform layer; $I N L$ : inner nuclear layer; $I P L$ : inner plexiform layer; $G C L$ : ganglion cell layer

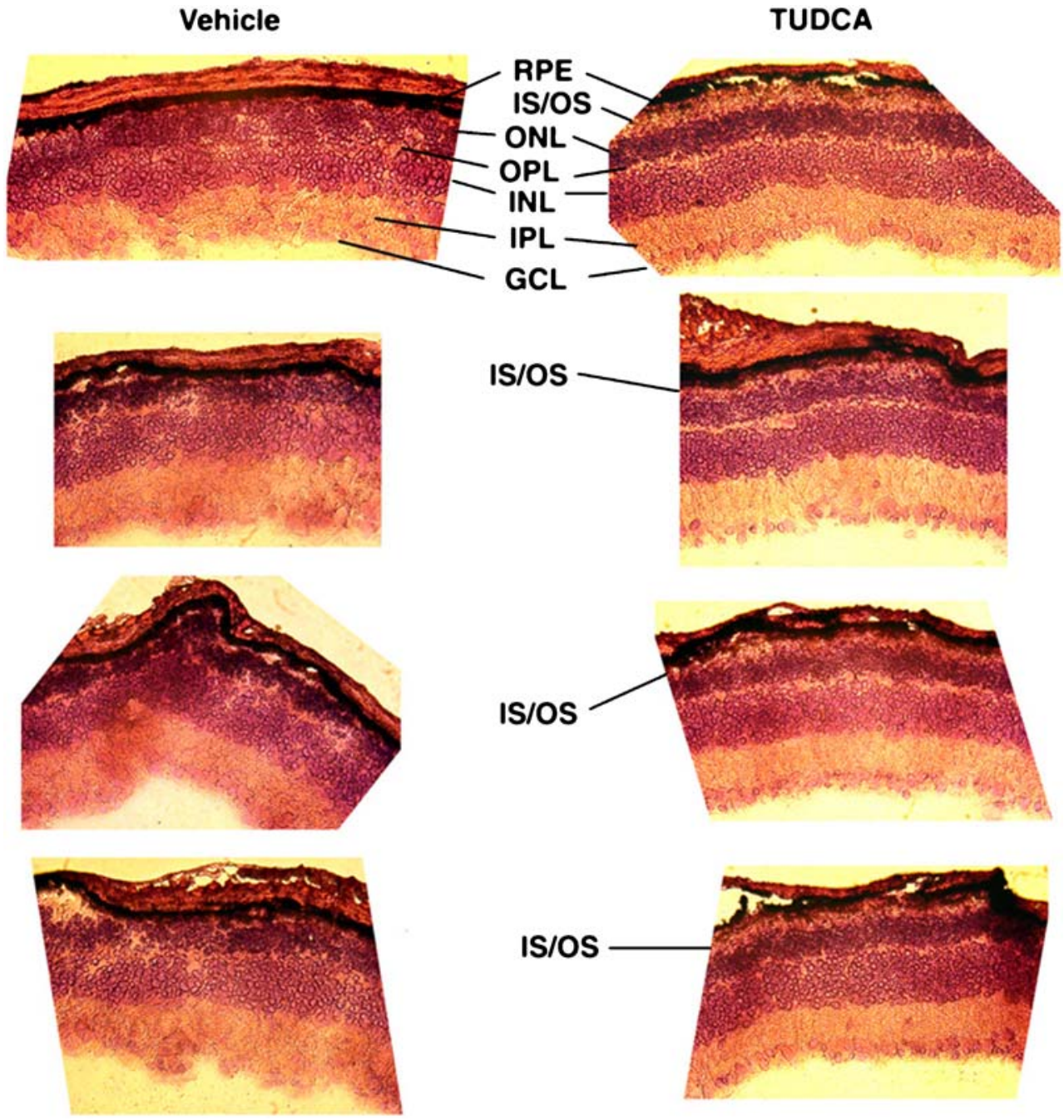




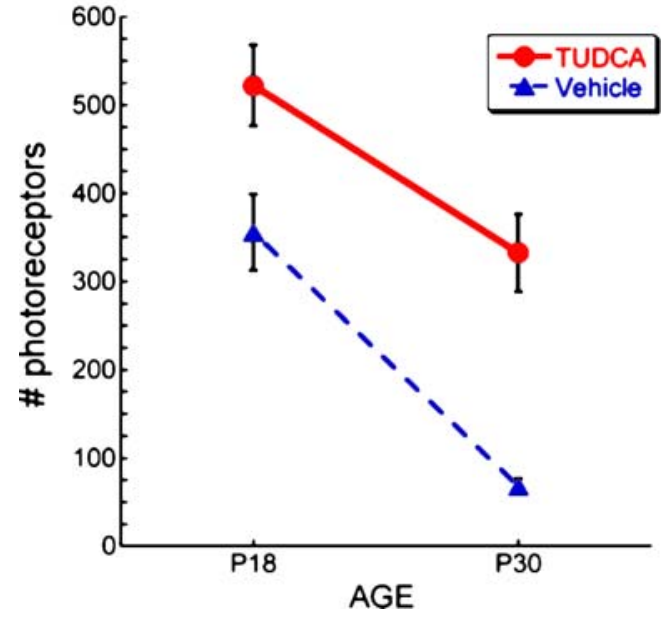

Fig. 2 Effect of TUDCA treatment on number of photoreceptor cells in P18 and P30 rd10 mice. rd10 were injected subcutaneously with vehicle or TUDCA $(500 \mathrm{mg} / \mathrm{kg})$ every 3 days starting at P6. At P18 and P30 mice were killed, and retinal paraffin sections were prepared and H\&E-stained. Outer nuclear layer nuclei were counted in two regions per section and were assumed to reflect number of photoreceptor cells. Each region spanned $400 \mu \mathrm{m}$ starting from a point $400 \mu \mathrm{m}$ from either side of the optic nerve. TUDCA treatment significantly preserved the number of photoreceptors at both postnatal days. Of note is that TUDCA treatment delayed the loss of photoreceptor cells by 12 days over the course of the degeneration to P30

wave amplitudes (Fig. 4 and [81]). Retina sections from mice treated with TUDCA showed almost no TUNEL signal (Fig. 3) and substantially reduced immunosignal for activated caspase 3 (Fig. 5), suggesting that treatment resulted in suppression of apoptosis [81].
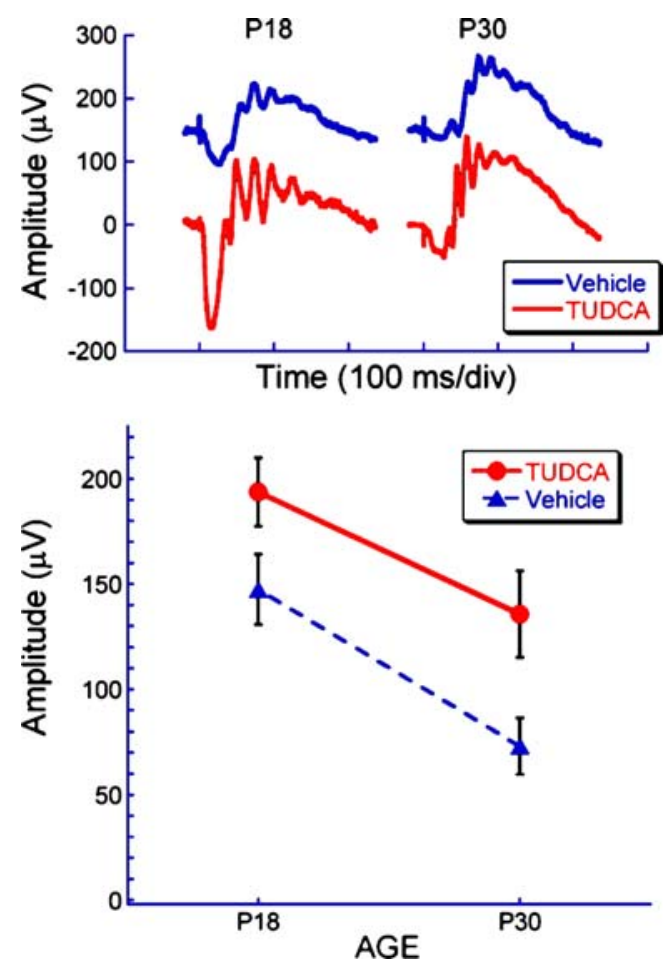

Fig. 4 Effect of TUDCA treatment on electroretinograms of rd10 mice at P18 and P30. Representative dark-adapted ERG waveforms (top) and average b-wave amplitude (bottom) to a bright flash (2.1 log $\left.\mathrm{cd} \mathrm{s} / \mathrm{m}^{2}\right)$ at P18 and P30 in TUDCA and vehicle-treated rd10 mice. Note that TUDCA treatment delayed the loss of retinal function by about 12 days, or about $35 \%$, over the course of the degeneration period (e.g., P30 TUDCA-treated amplitudes are similar to P18 vehicle-treated amplitudes)

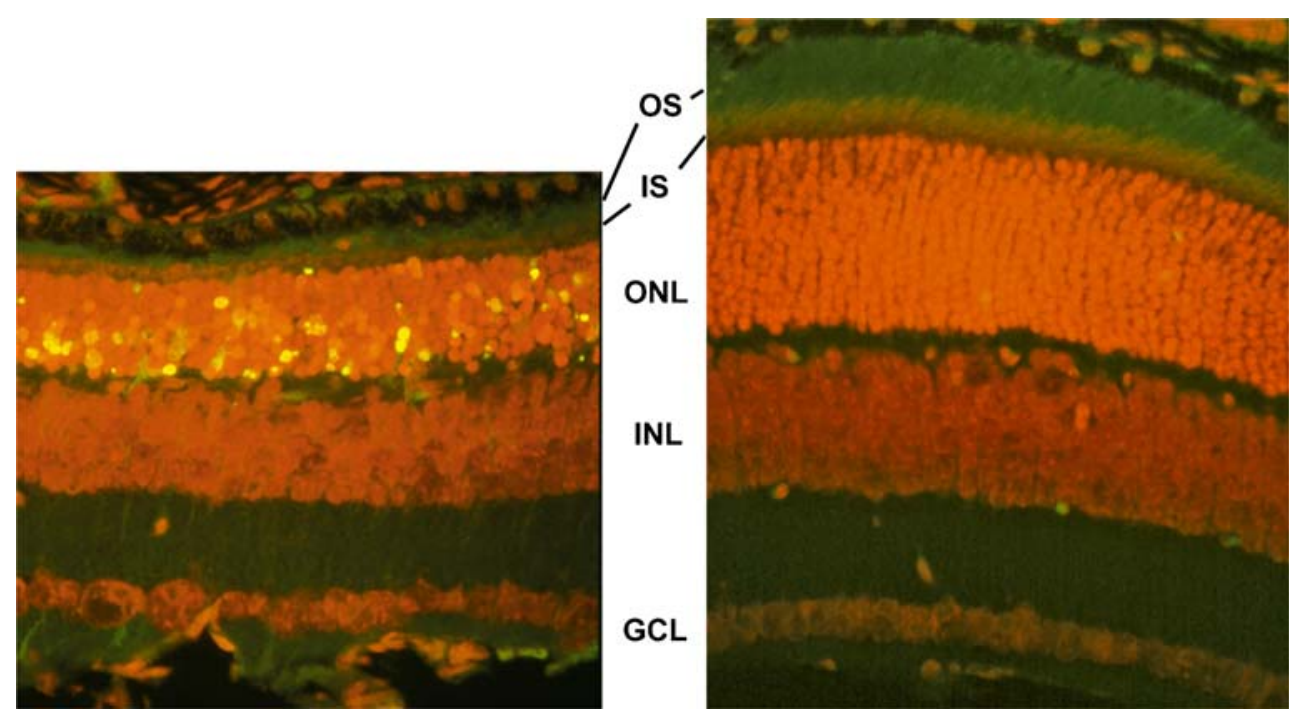

Fig. 3 Effect of TUDCA treatment on retinal morphology and TUNEL in P18 rd10 mice. Fluorescence microscopy using a B-2A emission fluorescence filter allows observation of the preservation of photoreceptor inner segments $(I S)$ and outer segments $(O S)$ present in TUDCA- (right) versus vehicle-treated retina sections (left). TUNELpositive nuclei (green/yellow signal) are seen to be abundant in

vehicle-treated sections, but rare retinas from TUDCA-treated mice. TUDCA treatment provided significant preservation of photoreceptor nuclei number in the outer nuclear layer $(O N L)$. Treatment had no discernable effect on the inner nuclear layer $(I N L)$ or ganglion cell layer $(G C L)$. Image reprinted with permission from Ref. [81] 
Fig. 5 Effect of TUDCA on caspase-3 activation in $\mathrm{rd} 10$ mouse retina. Paraffinembedded rd10 mouse retina sections from mice treated with vehicle or TUDCA were assayed for immunoreactivity to activated caspase- 3 and observed by confocal microscopy. There was significantly more immunoreactivity (yellow signal) in vehicletreated sections than in

TUDCA-treated sections. Image reprinted with permission from Ref. [81]

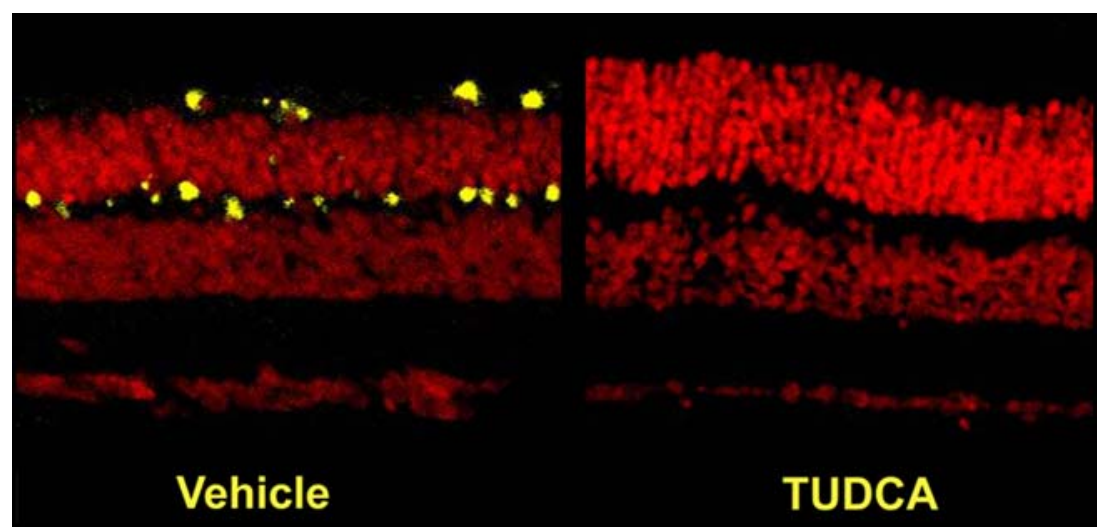

Systemic TUDCA treatment provides protection in the rd10 mouse retina to at least P30. At this stage, the ONL has degenerated to about one cell layer of mainly cones (rods are nearly completely absent and cones are degenerating), and the dark-adapted a-wave is only $3 \%$ and the bwave only $14 \%$ of wild-type mice $[80,82]$. TUDCA-treated retinas had dark-adapted a-waves that were maintained to $30 \%$ of wild-type and light- and dark-adapted b-waves maintained to $45 \%$ of wild-type, indicating preservation of both rod and cone function (Fig. 4 and [82]). The number of photoreceptor nuclei was fivefold greater in TUDCAtreated mice than in vehicle-treated mice (Figs. 2 and 6). Of

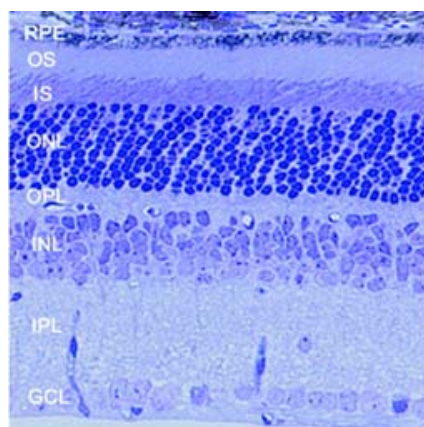

a

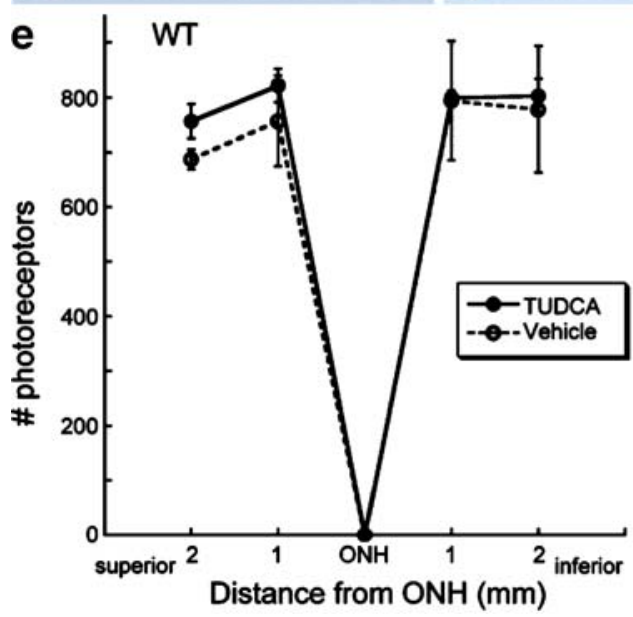

Fig. 6 Effect of TUDCA treatment on retinal morphology in rd10 and wild-type mice at P30. Retinal micrographs from P30 wild-type C57BL/6 mice treated with TUDCA (a) or vehicle (b) and from rd10 mice treated with TUDCA (c) or vehicle (d). TUDCA treatment has no effect on wild-type retinal morphology. In $\mathrm{rd} 10$ mice, the photoreceptor layer has been reduced to approximately one row of nuclei (d), but TUDCA treatment preserved three to four rows of nuclei (c). $\mathbf{c}$ and $\mathbf{d}$ Clear differences in the thicknesses of the ONL,
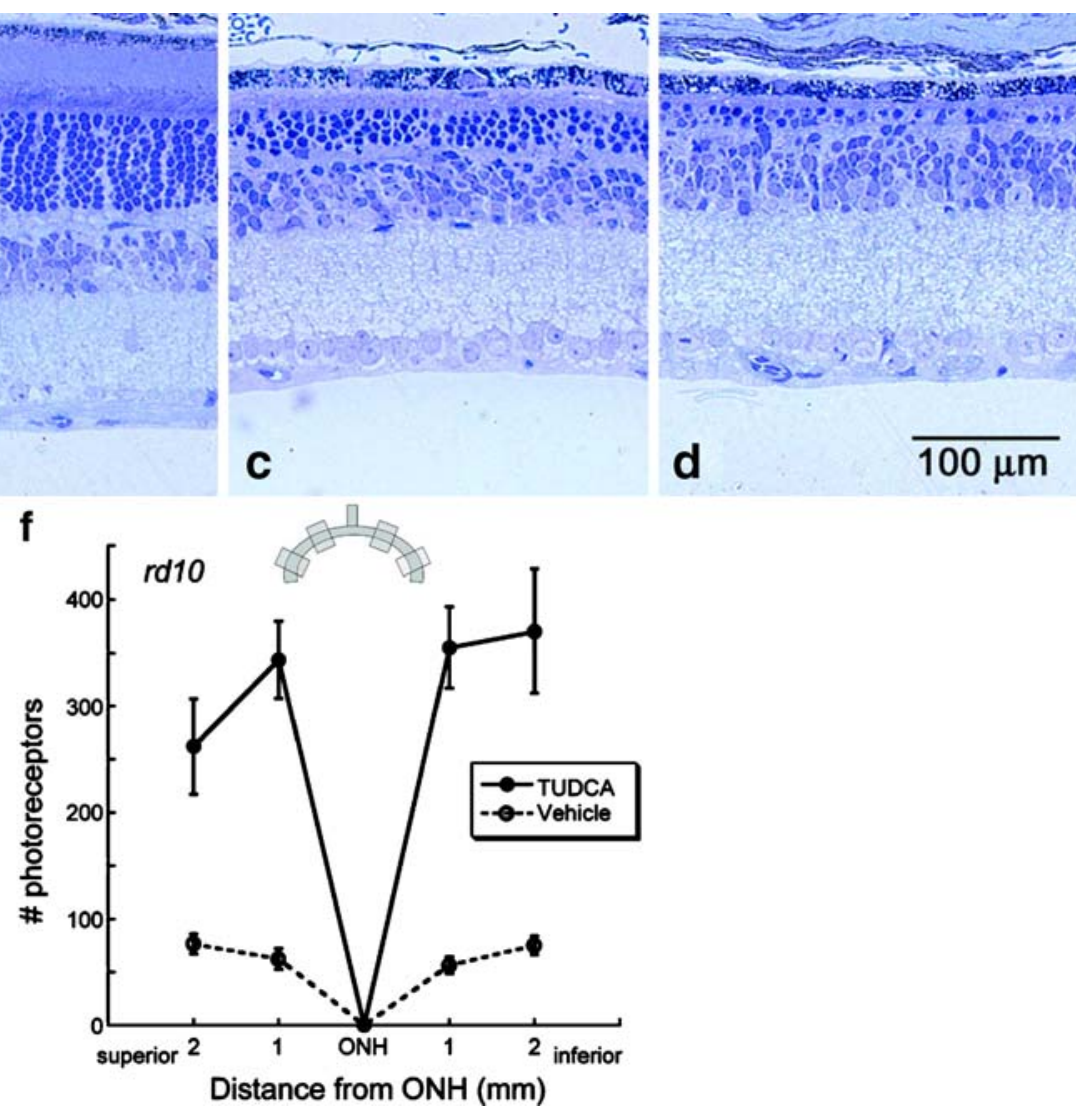

outer segments $(O S)$, and inner segments $(I S)$, with TUDCA treatment demonstrating a delay of retinal degeneration. $\mathbf{e}$ and $\mathbf{f}$ Plots of the total number of photoreceptors at each retinal location from wild type and rd10 mice with reference to the optic nerve head $(O N H)$. The inset is a diagram of the retina and optic nerve and the areas from which cells numbers were sampled. The TUDCA-treated mice have significantly more photoreceptors across all areas sampled than vehicle-treated mice. Image reprinted from Ref. [82] 
note is that TUDCA treatment delayed the loss of photoreceptor cell number and retinal function by 12 days over the course of the degeneration to P30 (Figs. 2 and 4). Similar to the effect on rod photoreceptors at P18, treatment preserved cone outer segment morphology in the P30 retina [82].

Other researchers have tested the effects of TUDCA in retinal degeneration models. TUDCA treatment slows retinal degeneration in s334ter-3 rats [83], a rat line that was genetically engineered to have a rhodopsin mutation identical to one common in autosomal dominant retinitis pigmentosa (ADRP) patients [84]. These rats were injected daily from birth with TUDCA. Retinal sections from P5 and P10 rats showed that TUDCA treatment significantly decreased markers for reactive oxygen species, endoplasmic reticulum (ER) stress, and apoptosis. Retinal degeneration as assessed by morphology was also delayed in TUDCA-treated rats [83].

TUDCA treatment also slows retinal degeneration in P23H-3 rats $[85,86]$, another rat line that was genetically engineered to have an ADRP rhodopsin mutation [84]. Rats were injected intraperitoneally $(500 \mathrm{mg} / \mathrm{kg}$ body weight) once per week from P20 through 4 months old. In TUDCAversus vehicle-treated rats, photoreceptor inner and outer segments, nuclei counts in the ONL, and the capillary retinal network were preserved. TUNEL signal was lower in TUDCA-treated rats compared to controls.

\section{Induced models of photoreceptor degeneration}

We also tested the effects of TUDCA treatment in mice and rats undergoing light-induced retinal degeneration (LIRD), an environmental model of blindness [87, 88]. Adult albino $\mathrm{BALB} / \mathrm{C}$ mice were subcutaneously injected with TUDCA or vehicle, dark-adapted for $18 \mathrm{~h}$, injected again, then exposed to $7 \mathrm{~h}$ of bright $(10,000 \mathrm{~lx})$ or $\operatorname{dim}(200 \mathrm{~lx})$ light [87], then returned to regular rearing lighting conditions. At various times post-exposure, ERGs, retinal morphology, and apoptosis markers were assessed. Within $24 \mathrm{~h}$ of damaging light exposure, there was massive disruption of photoreceptor cells, extreme disorganization, and heavy TUNEL signal throughout the ONL in vehicle-treated mice exposed to bright light (Fig. 7). Conversely, retinas from TUDCA-treated mice exposed to bright light had low TUNEL signal and unaltered retinal morphology (Fig. 7), similar to mice exposed to dim light. Immunoreactivity for activated caspase- 3 was abundant in retina sections from vehicle-injected mice, but nearly nonexistent in sections from TUDCA-injected mice exposed to bright light or mice exposed to dim light (Fig. 8). Protection against light damage was observed at 15 days post-exposure, again, with suppression of TUNEL (Fig. 9) and diminished immunosignal for activated caspase 3 [81]. ERG amplitudes were maintained in TUDCA-treated mice exposed to bright light, even up to 7 weeks post-exposure [89], suggesting that protection is fairly long term. Thus, in both the rd10 mouse and the LIRD mouse and rat, apoptotic photoreceptor degeneration was greatly diminished and retinal function was largely preserved by systemic injections of TUDCA.

Ganglion cell death model

We also found that systemic TUDCA treatment slowed loss of retinal ganglion cells following optic nerve crush. Adult C57/B16 mice were treated as in the LIRD and rd10 mouse experiments for 10 days and anesthetized, and their optic nerves were transected [90, 91]. In some mice, a gelfoam sponge soaked in 3\% Fluorogold was applied to the cut optic nerve stumps. Ten days after surgery, the number of fluorescing RGC somas seen in retina flatmounts declined by about $60 \%$ in vehicle-treated animals, a loss that was completely prevented in TUDCA-treated mice [90].

\section{Lens models}

Thus, systemic treatment with TUDCA slows retinal degeneration in mouse and rat models, but it also has effects in other ocular tissues. Shinohara and colleagues report that TUDCA treatment prevents lens epithelial cell (LEC) death and cataract formation [92]. Incubating

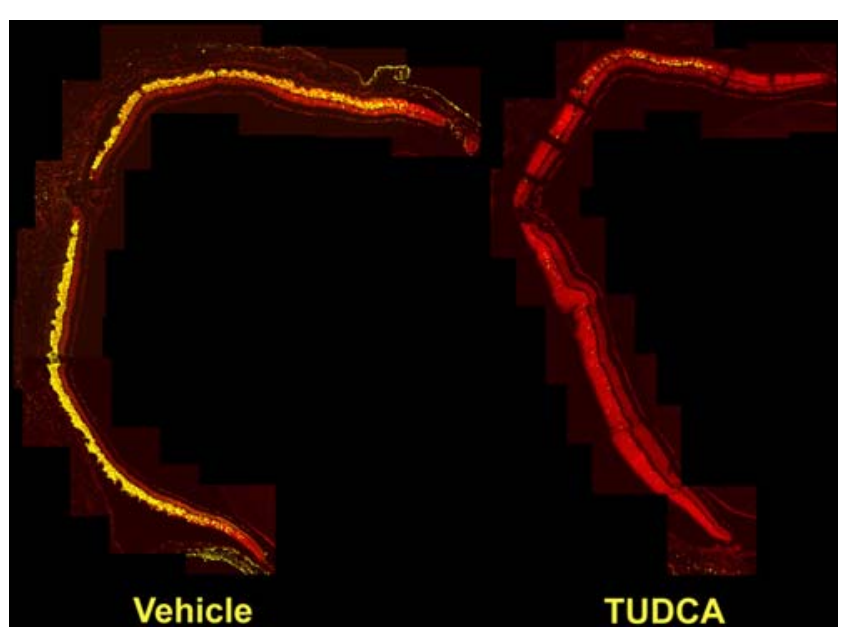

Fig. 7 Effect of TUDCA on LIRD mouse retina morphology and apoptosis: 24-h post-light exposure. Mice were subcutaneously injected with either vehicle or TUDCA $(500 \mathrm{mg} / \mathrm{kg})$, exposed to $10,000 \mathrm{~lx}$ of white light for $7 \mathrm{~h}$, then returned to maintenance lighting conditions. Mice were killed $24 \mathrm{~h}$ later, and paraffin retina sections were prepared and assayed for fluorescent TUNEL by confocal microscopy. Representative composite confocal micrographs for vehicle (left) versus TUDCA (right) treatments are shown. Brightlight-exposure-induced massive apoptosis (yellow signal) and morphological damage in photoreceptor cells and retinal outer nuclear layer of vehicle- (left) but not TUDCA-treated eyes (right). Image reprinted with permission from Ref. [81] 


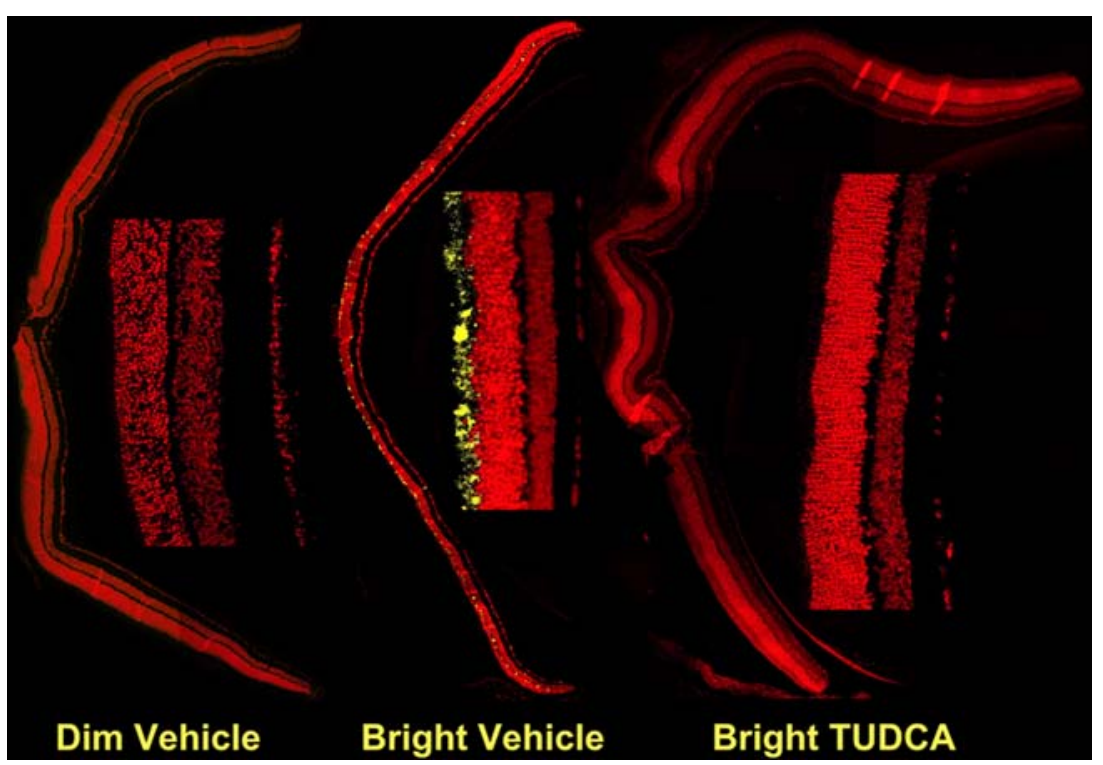

Fig. 8 Effect of TUDCA on LIRD mouse retina caspase-3 activation. Mice were subcutaneously injected with either vehicle or TUDCA $(500 \mathrm{mg} / \mathrm{kg})$, exposed to 200 (dim) or 10,000 lx (bright) of white light for $7 \mathrm{~h}$, then returned to maintenance lighting conditions. Mice were killed $24 \mathrm{~h}$ later, and paraffin retina sections were prepared and probed for activated caspase 3 immunoreactivity by fluorescent confocal microscopy. Representative composite confocal micrographs for vehicle dim-light-exposed vehicle-injected (left), bright-light-exposed vehicle-injected, and bright-light exposed TUDCA-injected (right) treatments are shown. There was significantly more immunoreactivity (yellow signal) in sections of bright-light-exposed vehicle-treated mice than in bright-light-exposed TUDCA-treated mice or dim-lightexposed mice. Image reprinted with permission from Ref. [81]

hypermature cataract formation in galactosemic rats, an effect accompanied by suppression of ER stress markers [92]. Though others have reported that UDCA and TUDCA efficacy is mediated through alleviation of ER stress [93-96], only Shinohara and colleagues have demonstrated that TUDCA acts as a chemical ER stress chaperone [83, 97].

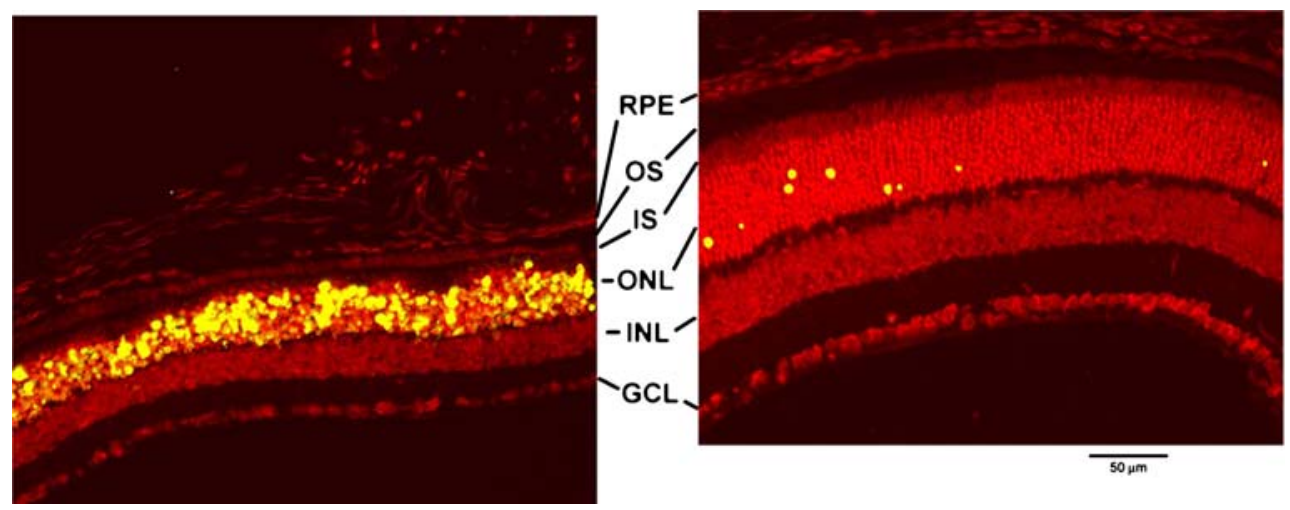

Fig. 9 Effect of TUDCA on LIRD mouse retina morphology and apoptosis: 15 days post-light exposure. Mice were subcutaneously injected with either vehicle or TUDCA $(500 \mathrm{mg} / \mathrm{kg})$, exposed to $10,000 \mathrm{~lx}$ of white light for $7 \mathrm{~h}$, then returned to maintenance lighting conditions. Fifteen days after light exposure, mice were killed, and paraffin retina sections were prepared and assayed for fluorescent TUNEL by confocal microscopy. Bright-light-exposure-induced massive apoptosis and morphological damage in retinal photoreceptors of vehicle-treated (left) but not TUDCA-treated eyes (right). Of particular note in the vehicle-treated sample is the thinning of the outer nuclear layer $(O N L)$ and the nearly complete loss of inner segments $(I S)$ and outer segments $(O S)$ of the photoreceptors. Additionally, nearly all the photoreceptor nuclei are TUNEL positive. Conversely, TUDCA-treated samples showed intact photoreceptors, thick ONL, and very few TUNEL-positive photoreceptor cells. Treatment had no discernable effect on the inner nuclear layer $(I N L)$, ganglion cell layer $(G C L)$, or retinal pigment epithelium (RPE). Image reprinted with permission from Ref. [81] 


\section{Conclusion}

The hydrophilic bile acids UDCA and TUDCA are cytoprotective in many disease models. Their exact mechanisms of action at the molecular level are not fully elucidated. Their cytoprotective actions are proposed to be mediated through actions at several points of the intrinsic and extrinsic apoptotic pathways and the ER stress response $[42,43,92,96,98]$ from direct actions on mitochondrial and ER membranes through transcriptional regulation of both death and survival factors. Effects in ocular disease models have just begun to be tested and are clearly promising. However, almost no mechanistic data from ocular models have been reported. Indeed, some of the protective effects of TUDCA may be due to its taurine moiety suppressing formation of the apoptosome $[99,100]$ ! Thus, in addition to continued preclinical testing for translational purposes, much work remains in determining how the hydrophilic bile acids have such marked protective effects in models of retinal degeneration, glaucoma, and cataract.

Open Access This article is distributed under the terms of the Creative Commons Attribution Noncommercial License which permits any noncommercial use, distribution, and reproduction in any medium, provided the original author(s) and source are credited.

\section{References}

1. Cidian Z. Dictionary of traditional Chinese medicine. Shanghai: Shanghai Science and Technology Press; 2004.

2. Lee Y-J. The use of bear bile as medicine versus tonic. In: Williamson DF and Phipps MJ, editors. Proceedings of the Third International Symposium on the Trade in Bear Parts. Seoul: TRAFFIC East Asia; 1999. p. 122-126

3. Feng Y, Siu K, Wang N, Ng KM, Tsao SW, Nagamatsu T, et al. Bear bile: dilemma of traditional medicinal use and animal protection. J Ethnobiol Ethnomed. 2009;5:2.

4. Gabriel GG.A bitter medicine: the use of bear bile in China. In: Williamson DF and Phipps MJ, editors. Proceedings of the Third International Symposium on the Trade in Bear Parts. Seoul: TRAFFIC East Asia; 1999. p. 116-120.

5. Hanson DG. Poaching: Oriental demand for undgam and paws decimates California bears. Audubon. 1983;85:127-8.

6. Comer C, Dixon J (2004) Illegal trade in whole black bears and black bear parts (Fisheries, S.N.P.a.V.D.o.G.a.I., eds). National Park Service, U.S. Department of the Interior

7. Raloff J. A galling business. Sci News. 2005;168:250-2.

8. Fund WW (2009) Wildlife Trade. http://www.cites.org/eng/app/ index.shtml

9. Congress, U.S. (1973). ENDANGERED SPECIES ACT OF 1973 As Amended through the 108th Congress

10. Mills J, Servheen C. The Asian trade in bears and bear parts: impacts and conservation recommendations. Bears: Their Biology and Management. 1994;9:161-7.

11. Hofmann AF. The continuing importance of bile acids in liver and intestinal disease. Arch Intern Med. 1999;159:2647-58.

12. Jones JD, Zollman PE. Black bear (Ursus americanus) bile composition: seasonal changes. Comp Biochem Physiol C Pharmacol Toxicol Endocrinol. 1997;118:387-90.
13. Hagey LR, Crombie DL, Espinosa E, Carey MC, Igimi H, Hofmann AF. Ursodeoxycholic acid in the Ursidae: biliary bile acids of bears, pandas, and related carnivores. J Lipid Res. 1993;34:1911-7.

14. Sola S, Garshelis DL, Amaral JD, Noyce KV, Coy PL, Steer CJ, et al. Plasma levels of ursodeoxycholic acid in black bears, Ursus americanus: seasonal changes. Comp Biochem Physiol C Toxicol Pharmacol. 2006;143:204-8.

15. MacDonald AC, Williams CN. Studies of bile lipids and bile acids of wild North American black bears in Nova Scotia, showing a high content of ursodeoxycholic acid. J Surg Res. 1985;38:173-9.

16. Bentayeb K, Batlle R, Sanchez C, Nerin C, Domeno C. Determination of bile acids in human serum by on-line restricted access material-ultra high-performance liquid chromatographymass spectrometry. J Chromatogr B Analyt Technol Biomed Life Sci. 2008;869:1-8.

17. Kanazawa T, Shimazaki A, Sato T, Hoshino T. Syntheses of ursodesoxycholic acid and its conjugated bile acid. Proceedings of the Japanese Academy. 1954;30:391-4.

18. Crosignani A, Battezzati PM, Setchell KD, Invernizzi P, Covini $\mathrm{G}$, Zuin M, et al. Tauroursodeoxycholic acid for treatment of primary biliary cirrhosis. A dose-response study. Dig Dis Sci. 1996;41:809-15.

19. Bachrach WH, Hofmann AF. Ursodeoxycholic acid in the treatment of cholesterol cholelithiasis. PART I. Dig Dis Sci. 1982;27:737-61.

20. Ward A, Brogden RN, Heel RC, Speight TM, Avery GS. Ursodeoxycholic acid: a review of its pharmacological properties and therapeutic efficacy. Drugs. 1984;27:95-131.

21. Batta AK, et al. Effect of long-term treatment with ursodiol on clinical and biochemical features and biliary bile acid metabolism in patients with primary biliary cirrhosis. Am J Gastroenterol. 1993;88:691-700.

22. Oka H, et al. A multi-center double-blind controlled trial of ursodeoxycholic acid for primary biliary cirrhosis. Gastroenterol Jpn. 1990;25:774-80.

23. Parquet M, Metman EH, Raizman A, Rambaud JC, Berthaux N, Infante R. Bioavailability, gastrointestinal transit, solubilization and faecal excretion of ursodeoxycholic acid in man. Eur J Clin Invest. 1985;15:171-8.

24. Rubin RA, Kowalski TE, Khandelwal M, Malet PF. Ursodiol for hepatobiliary disorders. Ann Intern Med. 1994;121:207-18.

25. Stiehl A, et al. Ursodeoxycholic acid-induced changes of plasma and urinary bile acids in patients with primary biliary cirrhosis. Hepatology. 1990;12:492-7.

26. Fedorowski T, Salen G, Calallilo A, Tint GS, Mosbach EH, Hall JC. Metabolism of ursodeoxycholic acid in man. Gastroenterology. 1977;73:1131-7.

27. Hofmann AF. Pharmacology of ursodeoxycholic acid, an enterohepatic drug. Scand J Gastroenterol Suppl. 1994;204:1-15.

28. Nakagawa M, Colombo C, Setchell KD. Comprehensive study of the biliary bile acid composition of patients with cystic fibrosis and associated liver disease before and after UDCA administration. Hepatology. 1990;12:322-34.

29. Paumgartner G, Beuers U. Ursodeoxycholic acid in cholestatic liver disease: mechanisms of action and therapeutic use revisited. Hepatology. 2002;36:525-31.

30. Tint GS, Salen G, Colalillo A, Graber D, Verga D, Speck J, et al. Ursodeoxycholic acid: a safe and effective agent for dissolving cholesterol gallstones. Ann Intern Med. 1982;97:351-6.

31. Walker S, Rudolph G, Raedsch R, Stiehl A. Intestinal absorption of ursodeoxycholic acid in patients with extrahepatic biliary obstruction and bile drainage. Gastroenterology. 1992;102:810-5.

32. Setchell KD, Rodrigues CM, Podda M, Crosignani A. Metabolism of orally administered tauroursodeoxycholic acid in patients with primary biliary cirrhosis. Gut. 1996;38:439-46. 
33. Salen G, Tint GS, Shefer S. Treatment of cholesterol gallstones with litholytic bile acids. Gastroenterol Clin North Am. 1991;20:171-82.

34. Saksena S, Tandon RK. Ursodeoxycholic acid in the treatment of liver diseases. Postgrad Med J. 1997;73:75-80.

35. Leuschner U, Leuschner M, Sieratzki J, Kurtz W, Hubner K. Gallstone dissolution with ursodeoxycholic acid in patients with chronic active hepatitis and two years follow-up. A pilot study. Dig Dis Sci. 1985;30:642-9.

36. Koga H, Sakisaka S, Ohishi M, Sata M, Tanikawa K. Nuclear DNA fragmentation and expression of Bcl-2 in primary biliary cirrhosis. Hepatology. 1997;25:1077-84.

37. Rodrigues CM, Fan G, Wong PY, Kren BT, Steer CJ. Ursodeoxycholic acid may inhibit deoxycholic acid-induced apoptosis by modulating mitochondrial transmembrane potential and reactive oxygen species production. Mol Med. 1998;4:165-78.

38. Rodrigues CM, Ma X, Linehan-Stieers C, Fan G, Kren BT, Steer CJ. Ursodeoxycholic acid prevents cytochrome c release in apoptosis by inhibiting mitochondrial membrane depolarization and channel formation. Cell Death Differ. 1999;6:842-54.

39. Rodrigues CM, Sola S, Sharpe JC, Moura JJ, Steer CJ. Tauroursodeoxycholic acid prevents Bax-induced membrane perturbation and cytochrome $\mathrm{C}$ release in isolated mitochondria. Biochemistry. 2003;42:3070-80.

40. Arenas F, Hervias I, Uriz M, Joplin R, Prieto J, Medina JF. Combination of ursodeoxycholic acid and glucocorticoids upregulates the AE2 alternate promoter in human liver cells. J Clin Invest. 2008;118:695-709.

41. Weitzel C, Stark D, Kullmann F, Scholmerich J, Holstege A, Falk W. Ursodeoxycholic acid induced activation of the glucocorticoid receptor in primary rat hepatocytes. Eur J Gastroenterol Hepatol. 2005;17:169-77.

42. Amaral JD, Viana RJ, Ramalho RM, Steer CJ, Rodrigues CM (2009). Bile acids: regulation of apoptosis by ursodeoxycholic acid. J Lipid Res 50:1721-1734

43. Sola S, Aranha MM, Steer CJ, Rodrigues CM. Game and players: mitochondrial apoptosis and the therapeutic potential of ursodeoxycholic acid. Curr Issues Mol Biol. 2007;9:123-38.

44. Rodrigues CM, Stieers CL, Keene CD, Ma X, Kren BT, Low $\mathrm{WC}$, et al. Tauroursodeoxycholic acid partially prevents apoptosis induced by 3-nitropropionic acid: evidence for a mitochondrial pathway independent of the permeability transition. J Neurochem. 2000;75:2368-79.

45. Keene CD, Rodrigues CM, Eich T, Linehan-Stieers C, Abt A, Kren BT, et al. A bile acid protects against motor and cognitive deficits and reduces striatal degeneration in the 3-nitropropionic acid model of Huntington's disease. Exp Neurol. 2001;171:351-60.

46. Davies SW, et al. Formation of neuronal intranuclear inclusions underlies the neurological dysfunction in mice transgenic for the HD mutation. Cell. 1997;90:537-48.

47. Mangiarini L, et al. Exon 1 of the HD gene with an expanded CAG repeat is sufficient to cause a progressive neurological phenotype in transgenic mice. Cell. 1996;87:493-506.

48. Viana RJ, et al. Tauroursodeoxycholic acid prevents E22Q Alzheimer's Abeta toxicity in human cerebral endothelial cells. Cell Mol Life Sci. 2009;66:1094-104.

49. Ramalho RM, Borralho PM, Castro RE, Sola S, Steer CJ, Rodrigues CM. Tauroursodeoxycholic acid modulates p53mediated apoptosis in Alzheimer's disease mutant neuroblastoma cells. J Neurochem. 2006;98:1610-8.

50. Ramalho RM, Viana RJ, Low WC, Steer CJ, Rodrigues CM. Bile acids and apoptosis modulation: an emerging role in experimental Alzheimer's disease. Trends Mol Med. 2008;14:54-62.

51. Ramalho RM, Viana RJ, Castro RE, Steer CJ, Low WC, Rodrigues CM. Apoptosis in transgenic mice expressing the P301L mutated form of human tau. Mol Med. 2008;14:309-17.
52. Rodrigues CM, Sola S, Brito MA, Brondino CD, Brites D, Moura JJ. Amyloid beta-peptide disrupts mitochondrial membrane lipid and protein structure: protective role of tauroursodeoxycholate. Biochem Biophys Res Commun. 2001;281:468-74.

53. Sola S, Castro RE, Laires PA, Steer CJ, Rodrigues CM. Tauroursodeoxycholic acid prevents amyloid-beta peptideinduced neuronal death via a phosphatidylinositol 3-kinasedependent signaling pathway. Mol Med. 2003;9:226-34.

54. Joo SS, Won TJ, Lee DI. Potential role of ursodeoxycholic acid in suppression of nuclear factor kappa B in microglial cell line (BV-2). Arch Pharm Res. 2004;27:954-60.

55. Duan WM, Rodrigues CM, Zhao LR, Steer CJ, Low WC. Tauroursodeoxycholic acid improves the survival and function of nigral transplants in a rat model of Parkinson's disease. Cell Transplant. 2002;11:195-205.

56. Rodrigues CM, Sola S, Nan Z, Castro RE, Ribeiro PS, Low WC, et al. Tauroursodeoxycholic acid reduces apoptosis and protects against neurological injury after acute hemorrhagic stroke in rats. Proc Natl Acad Sci U S A. 2003;100:6087-92.

57. Rodrigues CM, Spellman SR, Sola S, Grande AW, LinehanStieers C, Low WC, et al. Neuroprotection by a bile acid in an acute stroke model in the rat. J Cereb Blood Flow Metab. 2002;22:463-71.

58. Castro RE, Sola S, Ramalho RM, Steer CJ, Rodrigues CM. The bile acid tauroursodeoxycholic acid modulates phosphorylation and translocation of bad via phosphatidylinositol 3-kinase in glutamate-induced apoptosis of rat cortical neurons. J Pharmacol Exp Ther. 2004;311:845-52.

59. Park IH, Kim MK, Kim SU. Ursodeoxycholic acid prevents apoptosis of mouse sensory neurons induced by cisplatin by reducing P53 accumulation. Biochem Biophys Res Commun. 2008;377:1025-30.

60. Colak A, Kelten B, Sagmanligil A, Akdemir O, Karaoglan A, Sahan E, et al. Tauroursodeoxycholic acid and secondary damage after spinal cord injury in rats. J Clin Neurosci. 2008;15:665-71.

61. Chazouilleres O, Ballet F, Legendre C, Bonnefis MT, Rey C, Chretien $\mathrm{Y}$, et al. Effect of bile acids on ischemia-reperfusion liver injury. J Hepatol. 1991;13:318-22.

62. Baiocchi L, et al. TUDCA prevents cholestasis and canalicular damage induced by ischemia-reperfusion injury in the rat, modulating PKCalpha-ezrin pathway. Transpl Int. 2008;21:792800.

63. Rolo AP, Oliveira PJ, Moreno AJ, Palmeira CM. Protection against post-ischemic mitochondrial injury in rat liver by silymarin or TUDC. Hepatol Res. 2003;26:217-24.

64. Ejiri S, Eguchi Y, Kishida A, Ishigami F, Kurumi Y, Tani T, et al. Cellular distribution of thrombomodulin as an early marker for warm ischemic liver injury in porcine liver transplantation: protective effect of prostaglandin $\mathrm{I} 2$ analogue and tauroursodeoxycholic acid. Transplantation. 2001;71:721-6.

65. Falasca L, et al. Protective role of tauroursodeoxycholate during harvesting and cold storage of human liver: a pilot study in transplant recipients. Transplantation. 2001;71:1268-76.

66. Ishigami F, Naka S, Takeshita K, Kurumi Y, Hanasawa K, Tani T. Bile salt tauroursodeoxycholic acid modulation of Bax translocation to mitochondria protects the liver from warm ischemiareperfusion injury in the rat. Transplantation. 2001;72:1803-7.

67. Hertl M, Hertl MC, Malago M, Broelsch CE. In vivo protection of the pig liver against ischemia/reperfusion injury by tauroursodeoxycholate. Langenbecks Arch Surg. 1999;384:461-6.

68. Ono T, Imai K, Kohno H, Uchida M, Takemoto Y, Dhar DK, et al. Tauroursodeoxycholic acid protects cholestasis in rat reperfused livers: its roles in hepatic calcium mobilization. Dig Dis Sci. 1998;43:2201-10.

69. Ono T, Nagasue N, Kohno H, Uchida M, Takemoto Y, Dhar DK, et al. Effect of tauroursodeoxycholic acid on bile flow and 
calcium excretion in ischemia-reperfusion injury of rat livers. J Hepatol. 1995;23:582-90.

70. Nowak G, Noren UG, Wernerson A, Marschall HU, Moller L, Ericzon BG. Enteral donor pre-treatment with ursodeoxycholic acid protects the liver against ischaemia-reperfusion injury in rats. Transpl Int. 2005;17:804-9.

71. Pergel A, Zengin K, Cercel A, Aki H, Kaya S. The effects of somatostatin and ursodeoxycholic acid in preventing the ischemic injury of the liver following Pringle maneuver in obstructive jaundice-rat model. Hepatogastroenterology. 2007;54:229-33.

72. Rivard AL, Steer CJ, Kren BT, Rodrigues CM, Castro RE, Bianco RW, et al. Administration of tauroursodeoxycholic acid (TUDCA) reduces apoptosis following myocardial infarction in rat. Am J Chin Med. 2007;35:279-95.

73. Rajesh KG, Suzuki R, Maeda H, Yamamoto M, Yutong X, Sasaguri S. Hydrophilic bile salt ursodeoxycholic acid protects myocardium against reperfusion injury in a PI3K/Akt dependent pathway. J Mol Cell Cardiol. 2005;39:766-76.

74. Guo R, Ma H, Gao F, Zhong L, Ren J. Metallothionein alleviates oxidative stress-induced endoplasmic reticulum stress and myocardial dysfunction. J Mol Cell Cardiol. 2009;47:228-37.

75. Lee WY, Han SH, Cho TS, Yoo YH, Lee SM. Effect of ursodeoxycholic acid on ischemia/reperfusion injury in isolated rat heart. Arch Pharm Res. 1999;22:479-84.

76. Arora SK., Faulkner A, Kim M, Ciavatta V, Pardue M. Tudca preserves cones in fast degenerating Rd1 mice. Invest Ophthalmol Vis Sci. 2009;50:E-Abstract 978.

77. Bowes C, Li T, Danciger M, Baxter LC, Applebury ML, Farber DB. Retinal degeneration in the rd mouse is caused by a defect in the beta subunit of rod cGMP-phosphodiesterase. Nature. 1990;347:677-80.

78. Chang B, Hawes NL, Hurd RE, Davisson MT, Nusinowitz S, Heckenlively JR. Retinal degeneration mutants in the mouse. Vision Res. 2002;42:517-25.

79. Gargini C, Terzibasi E, Mazzoni F, Strettoi E. Retinal organization in the retinal degeneration 10 (rd10) mutant mouse: a morphological and ERG study. J Comp Neurol. 2007;500:222-38.

80. Chang B, et al. Two mouse retinal degenerations caused by missense mutations in the beta-subunit of rod cGMP phosphodiesterase gene. Vision Res. 2007;47:624-33.

81. Boatright $\mathrm{JH}$, et al. Tool from ancient pharmacopoeia prevents vision loss. Mol Vis. 2006;12:1706-14.

82. Phillips MJ, et al. Tauroursodeoxycholic acid preservation of photoreceptor structure and function in the rd10 mouse through postnatal day 30. Invest Ophthalmol Vis Sci. 2008;49:2148-55.

83. Mulhern ML, Madson CJ, Thoreson W, Shinohara T. Chemical chaperones and TUDCA partially suppress degeneration of retinal photoreceptor cells in transgenic mutant rhodopsin S334ter-3 rats. Invest Ophthalmol Vis Sci. 2008;49:E-Abstract 2038.

84. Steinberg RH, et al. Transgenic rat models of inherited retinal degeneration caused by mutant opsin genes. Invest Ophthalmol Vis Sci. 1996;37:S698.

85. Fernandez-Sanchez L, Lax P, Esquiva G, Pinilla I, Martín-Niet J, Cuenca N. Loss of synaptic contacts in the retina is prevented by tauroursodeoxycholic acid (TUDCA) in transgenic $\mathrm{P} 23 \mathrm{H}$ rats. Invest Ophthalmol Vis Sci. 2009;50:E-Abstract 980.

86. Fernandez-Sanchez L, Pinilla I, Campello L, Idiope M, MartinNieto J, Cuenca N. Tauroursodeoxycholic acid (TUDCA) slows retinal degeneration in transgenic P23H Rats. Invest Ophthalmol Vis Sci. 2008; 49:E-Abstract 2195.

87. Chen L, Dentchev T, Wong R, Hahn P, Wen R, Bennett J, et al. Increased expression of ceruloplasmin in the retina following photic injury. Mol Vis. 2003;9:151-8.

88. Reme CE, Grimm C, Hafezi F, Marti A, Wenzel A. Apoptotic cell death in retinal degenerations. Prog Retin Eye Res. 1998; 17:443-64.

89. Yang ES, Kendall C, Premji SM, Boatright JH. Tauroursodeoxycholic acid (TUDCA) prevents loss of visual function in rats. Invest Ophthalmol Vis Sci. 2008;49:E-Abstract 4933.

90. Boatright, Boyd AP, Garcia-Valenzuela E. Effect of tauroursodeoxycholic acid on mouse retinal ganglion cell degeneration following optic nerve transection. Invest Ophthalmol Vis Sci. 2005;46:E-Abstract 3180.

91. Garcia-Valenzuela E, Rayanade R, Perales JC, Davidson CA, Hanson RW, Sharma SC. Axon-mediated gene transfer of retinal ganglion cells in vivo. J Neurobiol. 1997;32:111-22.

92. Mulhern ML, Madson CJ, Kador PF, Randazzo J, Shinohara T. Cellular osmolytes reduce lens epithelial cell death and alleviate cataract formation in galactosemic rats. Mol Vis. 2007;13:1397-405.

93. Chen Y, Liu CP, Xu KF, Mao XD, Lu YB, Fang L, et al. Effect of taurine-conjugated ursodeoxycholic acid on endoplasmic reticulum stress and apoptosis induced by advanced glycation end products in cultured mouse podocytes. Am J Nephrol. 2008;28:1014-22.

94. de Almeida SF, Picarote G, Fleming JV, Carmo-Fonseca M, Azevedo JE, de Sousa M. Chemical chaperones reduce endoplasmic reticulum stress and prevent mutant HFE aggregate formation. J Biol Chem. 2007;282:27905-12.

95. Ozcan U, Yilmaz E, Ozcan L, Furuhashi M, Vaillancourt E, Smith RO, et al. Chemical chaperones reduce ER stress and restore glucose homeostasis in a mouse model of type 2 diabetes. Science. 2006;313:1137-40.

96. Xie Q, Khaoustov VI, Chung CC, Sohn J, Krishnan B, Lewis $\mathrm{DE}$, et al. Effect of tauroursodeoxycholic acid on endoplasmic reticulum stress-induced caspase-12 activation. Hepatology. 2002;36:592-601.

97. Shinohara T, Liang J, Song S, Mulhern ML, Thurston G. Chemical chaperones and bile acids protect molecular chaperones, alpha-crystallins against thermal denaturation in vitro. Invest Ophthalmol Vis Sci. 2008;49:E-Abstract 4504.

98. Ikegami T, Matsuzaki Y. Ursodeoxycholic acid: mechanism of action and novel clinical applications. Hepatol Res. 2008;38:123-31.

99. Takatani $\mathrm{T}$, et al. Taurine inhibits apoptosis by preventing formation of the Apaf-1/caspase-9 apoptosome. Am J Physiol Cell Physiol. 2004;287:C949-53.

100. Udawatte C, Qian H, Mangini NJ, Kennedy BG, Ripps H. Taurine suppresses the spread of cell death in electrically coupled RPE cells. Mol Vis. 2008;14:1940-50. 